Disclosure of Interests: None declared

DOI: 10.1136/annrheumdis-2019-eular.3956

\section{THU0728-HPR "I AM GOOD ENOUGH AS I AM EVEN IF I AM NOT PERFECT" -A QUALITATIVE STUDY OF FIBROMYALGIA PATIENTS' EXPERIENCES FROM PARTICIPATION IN A MINDFULNESS-BASED GROUP-PROGRAMME}

Heidi A. Zangi ${ }^{1}$, Gyda Singstad² Ingrid Ruud Knutsen ${ }^{2}$, Improved management of patients with fibromyalgia: Evaluation of an integrated care model. ${ }^{1}$ Diakonhjemmet Hospital, National Advisory Unit on Rehabilitation in Rheumatology, Oslo, Norway; ${ }^{2}$ Oslo Metropolitan University, Oslo, Norway

Background: People with fibromyalgia (FM) suffer from widespread pain, nonrefreshing sleep, fatigue and reduced mental wellbeing. No curative pharmacological treatment exists. Vitality Training (VTP) is a mindfulness- and acceptancebased group-programme that aims at enhancing participants' health promoting resources, strengthening their inner authority and ability to act according to own values. It combines mindfulness with creative methods and group counselling. Two RCTs have shown significant improvements in mental wellbeing, pain coping and fatigue in patients with chronic musculoskeletal pain and inflammatory arthritis. The VTP is currently being evaluated in an ongoing study for patients with newly diagnosed FM [1].

Objectives: The aim of this qualitative study was to explore FM patients' experiences from participating in the VTP, and if they perceived that it had any impact on their health and functioning.

Methods: Six qualitative in-depth interviews with participants from three VTPcourses were conducted following a semi-structured interview guide. Interviews were audio-recorded and transcribed. All three authors analysed the data by use of systematic text condensation.

Results: Three main themes were identified.

1. Understanding oneself in light of the group: Mutual understanding and acknowledgement had altered participants' self-understanding - from a feeling that "something was wrong with me" towards perceiving themselves as "a normal person with similar challenges as others".

2. Learning to accept oneself: Participants had obtained a greater understanding and acceptance for their emotions and reactions and had become kinder towards themselves. "I have realised how strict I have been towards myself... I am indeed good enough as I am even if I am not perfect".

3. Coping with everyday challenges: Becoming aware of what had provoked stress and learn how to face it had helped participants take more control. "I can do small changesthat really makes it better... the illness does not decide everything. Indeed I can decide something myself".

Conclusion: Participation in the VTP had contributed to new ways of relating to oneself and the illness. The support and acknowledgement participants experienced from the group had helped them alter their self-understanding from only being ill towards also being healthy and normal. They had learnt ways to better cope with stress and everyday challenges. Although this was a small study, the findings correspond with findings in previous studies.

\section{REFERENCES}

[1] Haugmark T. et al. Effects of a community-based multicomponent rehabilitation programme for patients with fibromyalgia: protocol for a randomised controlled trial. BMJ open. 2018 Jun 4; 8(6):e021004.

Disclosure of Interests: None declared

DOI: 10.1136/annrheumdis-2019-eular.3210

\section{HPR Professional education, training and competencies}

\section{THU0729-HPR BENEFITS OF EMPOWERING THE PATIENTS TO SELF- ADMINISTER SUBCUTANEOUS LOW DOSE METHOTREXATE (LD-MTX) INJECTIONS IN PATIENTS WITH SYSTEMIC IMMUNO-INFLAMMATORY RHEUMATIC DISEASES (SIRDS): A STUDY BY RHEUMATOLOGY NURSES COUNSELLOR}

Sadhana Baghel, Ravita Thakran, Christy Messi, Vlinay Yadav, Vivekanand Kashyap, Qamar Zaheer. Indian Spinal Injuries Centre, Rheumatology, New Delhi, India

Background: In recent years, early aggressive targeted treatment for SIRDs is recommended. LD-MTX is the "anchor drug" for these diseases. The administration of LD-MTX using subcutaneous route increases its therapeutic efficacy that ensures the maximum bioavailability and reduces gastrointestinal adverse effects. Therefore, it is preferred over the oral route. Increased use of subcutaneous self injection of medication has benefits for the patients and healthcare system. Increased use of subcutaneous self injection could alleviate pressure on medical services by reducing hospital/clinic visits. Therefore, explaining the benefits of self administration of subcutaneous injection such as early disease control, increased drug adherence, positive attitudes towards life, decreased financial burden of administration cost, decreased dependency to the others, should be an imperative part of patient's education.

Objectives: Benefits of self-administered subcutaneous LD-MTX injection in patients with SIRDs.

Methods: This retrospective study included patients who were prescribed injectable LD-MTX for their treatment. Besides the demographic information (age, gender) and disease characteristics (diagnosis, duration, prior treatment), the patients were interviewed and counselled regarding the virtues to take subcutaneous LD-MTX self-injection. The technique for selfinjection of LD-MTX subcutaneously was explained and demonstrated to the patients. All the information including the follow-up details, were recorded in a pre-designed form.

Results: Three hundred $(n=300)$ consecutive patients who were advised weekly LD-MTX injections and taught the self-injection technique were enrolled in this study. On follow-up visit it was found that among them, 177 patients $(59 \%)$ learned and started to administer their own subcutaneous injection of LD-MTX and they adhered to the injection schedule, in $50(16.7 \%)$ patients the injections was being given by the attendant (who had learnt the injection technique because the patients had deformities), while the other $73(24.3 \%)$ were not very compliant to injections due to psychological barriers towards self-injection, worried about pain and incorrect technique, adverse effects of the incorrect injections social stigma related to self-injections, and frustration or lack of acceptance of the illness.

Conclusion: By empowering the patients to self-administer LD-MTX injections subcutaneously, a majority $(59 \%)$ of them successfully continued to take the medicine appropriately. Thus this study shows the important role the nurses' play in educating patients and helping them to overcome psychological barriers to self injection. Empowering the patients to take selfadminister injections would result in better long term treatment adherence, improving treatment flexibility and overall quality of life.

\section{REFERENCES}

[1] Hazlewood, et al. The comparative effectiveness of oral versus subcutaneous methotrexate for the treatment of early rheumatoid arthritis. Ann Rheum Dis. May 2015

[2] Malaviya AN, Sharma A, Agarwal D, Kapoor S, Garg S, Sawhney S. Lowdose and high-dose methotrexate are two different drugs in practical terms. Int J Rheum Dis. 2010 Oct

Acknowledgement: The authors gratefully acknowledge the help and encouragement from Prof A. N. Malaviya HOD, Drs. Sanjiv Kapoor, SR Garg (Sr. Consultant Rheumatologists at ISIC. We also wish to thank Dr. Parth Sharma (Junior Resident), Mr. Himanshu Negi (Data Entry)

Disclosure of Interests: None declared

DOI: 10.1136/annrheumdis-2019-eular.1836 